\title{
Relationship of Th17/Treg Cells and Radiation Pneumonia in Locally Advanced Esophageal Carcinoma
}

\author{
YAN WANG ${ }^{1 *}$, GANG XU $^{1 *}$, JIE WANG $^{1}$, XIN-HUA LI ${ }^{2}$, PING SUN $^{2}$, \\ WEI ZHANG ${ }^{2}$, JUN-XIA LI ${ }^{2}$ and CHAO-YANG WU ${ }^{1}$ \\ ${ }^{1}$ Department of Radiation Oncology, Affiliated People's Hospital, Jiangsu University, Zhenjiang, P.R. China; \\ ${ }^{2}$ Department of Radiation Oncology, Yuhuangding Hospital of Qingdao University, Yantai, P.R. China
}

\begin{abstract}
Background/Aim: Radiation pneumonia is a main side-effect that has limited the clinical usage of radiotherapy in locally advanced esophageal carcinoma. Thelper cells 17 (Th 17) and T regulatory cells (Tregs) play an important role in inflammatory diseases. The balance between Treg and Th17 cells is a key factor in the progression of many inflammatory and autoimmune diseases. Whether Tregs and Th17 cells are predictive factors of radiation pneumonia has not yet been reported. In this study, we investigated the relationships of Treg/Th17 cells and radiation pneumonia in patients with locally advanced esophageal cancer who received radiotherapy. Patients and Methods: One hundred and forty-eight patients with locally advanced esophageal cancer who received radical and palliative radiotherapy were enrolled. The levels of Th17 and Treg cells in the blood of patients were detected using flow cytometry at the time point of pre-radiotherapy, 1st, 2nd, 3rd, 4th, 5th and 6th week from the start of radiation and 4 weeks after completion of radiotherapy. Radiation pneumonia was evaluated according to Radiation Therapy Oncology Group's acute radiation pneumonia standards, with the endpoint being grade 2 or above radiation pneumonia. Results: There were 24 cases of radiation pneumonia in 148 cases of locally
\end{abstract}

\footnotetext{
*These Authors contributed equally to this study.

Correspondence to: Jun-xia Li, MD, Department of Radiation Oncology, Yuhuangding Hospital of Qingdao University, NO.20 East Yuhuangding Road, Yantai, Shandong Province, P.R. China Tel: +86 13356994778, Fax: +86 53564240341, e-mail: lijunxia1972@163.com and Chao-yang Wu, MD, Department of Radiation Oncology, Affiliated People's Hospital, Jiangsu University, NO.8 Dianli Road, Zhenjiang, Jiangsu Province, P.R. China. Tel: +86 51188915043, Fax: +86 51185234387, e-mail: wuchaoyang9@163.com
}

Key Words: Esophageal cancer, radiotherapy, radiation pneumonia, Th17 cells, Treg cells. advanced esophageal cancer patients who underwent radiotherapy. Th17 cells increased and, in contrast, Treg cells decreased in the radiation pneumonia group. The change in the ratio of Th17/Treg was more pronounced and the difference was statistically significant from the 5th week after irradiation compared to patients with no radiation pneumonia $(p<0.05)$. There was no significant difference in dosimetric parameters, including V5, V2O, V30 and mean lung dose (MLD) and clinical factors, such as gender, age, smoking history, history of surgery and chemotherapy. Conclusion: The ratio of Th17/Treg cells may be an effective predictive factor of radiation pneumonia.

Radiation therapy is an effective treatment strategy for esophageal cancer (1-3). However, radiation pneumonia is a main side-effect that has limited the clinical usage of radiotherapy. Therefore, if radiation pneumonia can be predicted early enough, individualized dosage regimens that may improve radiation treatment outcome can be administered to patients.

Many studies show that some cytokines are closely related to radiation pneumonia, such as interleukin (IL)-6, IL-10, transforming growth factor (TGF)- $\beta 1$ (4-7). The most important cytokine is TGF- $\beta 1$, but NovakovaJiresova et al. reported that there is no significant difference in the concentration of serum TGF- $\beta 1$ between patients with and without radiation pneumonia (8-10). Therefore, TGF- $\beta 1$ is not a reliable predictive factor for the risk of radiation pneumonia. Recent studies have found that $\mathrm{T}$ helper cells 17 (Th17) and T regulatory cells (Tregs) play an important role in the inflammatory diseases. The balance between Tregs and Th17 cells is a key factor in the progress of many inflammatory and autoimmune diseases (11-13). Whether Treg cells and Th17 cells are predictive factors for radiation pneumonia has not yet been reported. In this article, we studied the predictive effect of Th17 and Treg cells on radiation pneumonia in locally advanced esophageal cancer. 


\section{Patients and Methods}

Patients. One hundred and forty-eight esophageal cancer patients treated with thoracic radiation at the Jiangsu University Affiliated People's Hospital between January 2010 and October 2014 were enrolled in this study. Inclusion criteria: (a) histological diagnosis of primary esophageal squamous cell carcinoma; (b) no previous thoracic radiotherapy; (c) dose-volume histogram (DVH) data available for analysis; (d) expected survival time >6 months; (e) Karnofsky performance score (KPS) $>70$.

Three dimensional radiotherapy planning. Radiotherapy was delivered through three-dimensional conformal radiotherapy using a linear accelerator (Siemens Oncor Berlin, Germany or Elekta, Stockholm, Sweden) with 6 MV or 10 MV X-Ray. All radiotherapy plans were designed using the Pinnacle 3 system. Simulation computed tomography (CT) scan was performed with the patients breathing freely and included the entire lung volume. The scan thickness was $3 \mathrm{~mm}$. Gross tumor volume (GTV) was defined as the gross tumor defined by the simulation $\mathrm{CT}$. The clinical target volume (CTV) included the GTV with $3.0-3.5 \mathrm{~cm}$ margin longitudinally and $1 \mathrm{~cm}$ axially. The planning target volume (PTV) was defined as CTV plus $0.5-1.0 \mathrm{~cm}$ margin in all directions. The prescribed dose was approximately $60-65 \mathrm{~Gy}, 1.8-2.0 \mathrm{~Gy}$ per fraction. Ninety-five percent volume of PTV received $100 \%$ of the prescribed dose. The spinal cord received a dose not more than 45 Gy and the mean dose received by the heart was not more than 30 Gy. No more than $30 \%$ of the combined lung volume was intended to receive more than $20 \mathrm{~Gy}$ (V20<30\%).

Flow cytometry analysis. Peripheral blood samples from the patients with locally advanced esophageal cancer were collected at the time point of pre-radiotherapy, 1st, 2nd, 3rd, 4th, 5th and 6th week from the start of radiation and 4 weeks after radiotherapy had been completed. Samples were then centrifuged at $1,000 \mathrm{rpm}$ for $5 \mathrm{~min}$, with the supernatant being discarded and added into the lymphocyte separation medium. Peripheral blood mononuclear cells were separated by density gradient centrifugation and seeded into 6-well plates at a concentration of $2 \times 10 \% / \mathrm{ml}$. CD4 ${ }^{+} \mathrm{IL}_{-17^{+}}$cells (Th17) and CD4+ CD25+Foxp3 cells (Tregs) were evaluated by flow cytometry analysis.

Follow-up and evaluation of radiation pneumonia. Early radiation pneumonia usually occurs 1 to 6 months after radiation, whereas late lung fibrosis usually occurs 6-24 months after radiation. Since we were interested in the prediction of early radiation pneumonia, a 4-month period as cut-off for diagnosis was used.

Patients were evaluated by radiation oncologists every two weeks during radiation and once a month until four months after radiotherapy. A chest CT scan was performed at each follow-up evaluation after completion of radiotherapy. Radiation pneumonia was scored according to the Radiation Therapy Oncology Group's acute radiation morbidity scoring criteria for pneumonia and the diagnosis of radiation pneumonia was based on the clinical symptoms and the corresponding radiographic changes in the radiation field.

Ethics statement. The study was approved by the Jiangsu University Affiliated People's Hospital ethics committee. All patients gave their consent for their information to be stored in the hospital database and subsequently used for research. All of the participants provided their written consent to participate in this study. Further, at the time of patient follow-up, in order to document the process, the presence of a qualified physician, a staff member of the Hospital ethics committee and a staff member of the medical records Department were required at the same time.

Statistical analysis. Data are represented as mean \pm standard deviation. Statistical analysis was performed using Pearson's $\chi^{2}$ test in the frequency tables or using one-way analysis of variance (ANOVA) in the comparison of the cell number of Th17 cells, Tregs and the ratio of Th17/Tregs between the two groups. In ANOVA, when a significant difference between groups was apparent, multiple comparisons of means were performed using the Dunnett test. All statistical assessments were two-sided and evaluated at the 0.05 level of significance. All statistical analyses were performed using SPSS 19.0 software (SPSS, Chicago, IL, USA).

\section{Results}

The incidence of radiation pneumonia. One hundred and forty-eight patients were included in this study with a median follow-up of 13 months (range=6-24 months). There were 24 patients $(16.2 \%)$ with grade 2 or above radiation pneumonia.

The relationship between clinical factors and radiation pneumonia. There was no significant relationship between clinical factors and radiation pneumonia. The clinical factors, including sex, age, smoking history, chemotherapy and thoracic surgery, are shown in Table I.

The relationship between $D V H$ parameters and radiation pneumonia. The lung dosimetric factors V5, V30 and mean lung dose (MLD) were calculated in patients with or without radiation pneumonia at grade 2 or above. The results are presented as mean \pm standard deviation. The results showed all dosimetric parameters were slightly higher in patients with than in patients without radiation pneumonia (Table II); however, there was a significant difference between the two groups $(p>0.05)$.

The relationship between Th17 cells, Treg and the ratio of Th17/Tregs and radiation pneumonia. Before radiotherapy, Th17 cells (the proportion of $\mathrm{CD}^{+}{ }^{+} \mathrm{T}$-cells) in the radiation pneumonia group and non-radiation pneumonia group were $2.59 \pm 1.11 \%$ and $2.51 \pm 1.21 \%$, respectively, whereas Treg cells were $0.72 \pm 4.19 \%$ and $4.13 \pm 1.02 \%$. There was no significant difference between the two groups. After the start of radiotherapy, Th17 cell numbers gradually increased; however, the Treg cell numbers decreased, so the Th17/Treg ratio further increased, and the changes in the radiation pneumonia group were more obvious. Four weeks after completion of radiotherapy, Th17 and Treg cells were more pronounced and there was a significant difference between the radiation pneumonia and non-radiation pneumonia groups (Figure 1A and B). The Th17/Treg ratio increased 
Table I. Relationship between clinical factors and radiation pneumonia.

\begin{tabular}{lcccc}
\hline Clinical factors & $\begin{array}{c}\text { Non-radiation } \\
\text { pneumonia } \\
(\mathrm{n}=124)\end{array}$ & $\begin{array}{c}\text { Radiation } \\
\text { pneumonia } \\
(\mathrm{n}=24)\end{array}$ & $\mathrm{x}^{2}$ & $p$-Value \\
\hline Gender & & & & \\
$\quad$ Male (78) & 64 & 14 & & \\
Female (70) & 60 & 10 & 0.364 & 0.546 \\
Age & & & & \\
$<60$ (38) & 32 & 6 & & \\
$\geq 60$ (110) & 92 & 18 & 0.007 & 0.934 \\
Smoking & & & & \\
Yes (92) & 75 & 17 & & \\
No (56) & 49 & 7 & 0.916 & 0.339 \\
Thoracic surgery & & & & \\
Yes (33) & 31 & 2 & 3.22 & 0.073 \\
No (115) & 93 & 22 & & \\
Chemotherapy & & 15 & & \\
Yes (89) & 74 & 9 & 0.067 & 0.796 \\
$\quad$ No (59) & 50 & & & \\
\hline
\end{tabular}

significantly in the radiation pneumonia group compared with the non-radiation pneumonia group at the fifth week after the start of radiotherapy (Figure 1C).

\section{Discussion}

Radiation-induced lung injury is a common side-effect in the radiotherapy of thoracic cancers (14-16), which reduces patients' quality of life and overall survival. Therefore, it would be advantageous to limit the clinical use of higher irradiation doses in the radiation course. Radiation pneumonia is affected by many factors, including physical factors (dose, irradiation area, V5, V20, V30, MLD) and clinical factors (age, gender, pulmonary function, smoking history, chemotherapy) (17-19). Many studies have shown that dosimetric parameters are closely related to radiation pneumonia, especially MLD $(20,21)$, which is significantly correlated to the occurrence of radiation pneumonia. Present existing data shows that when the dosimetric parameters are at the levels V10>34\%, V20>25\%, V30\%>18\%, MLD>15 $\mathrm{Gy}$, the incidence of radiation pneumonia will significantly increase. Among these parameters, V20 is thought of as the most important that, when controlled to less than $25 \%$, will reduce the incidence of radiation pneumonia. In our study, the dosimetric parameters V5, V20, V30, MLD were $60.4 \pm 5.7,26.2 \pm 4.8,16.4 \pm 4.5,15.8 \pm 4.1$, respectively, which is consistent with the results reported in the literature. However, there is no significant difference between the two groups in our study perhaps due to the small sample size.

Radiation pneumonia always occurs within one to three months after completion of radiotherapy, so some researches
Table II. Relationship between DVH parameters and radiation pneumonia.

\begin{tabular}{lcccc}
\hline & V5 & V20 & V30 & MLD \\
\hline Radiation pneumonia & $60.4 \pm 5.7$ & $26.2 \pm 4.8$ & $16.4 \pm 4.5$ & $15.8 \pm 4.1$ \\
Non-radiation pneumonia & $58.9 \pm 6.2$ & $24.2 \pm 6.0$ & $14.9 \pm 5.4$ & $14.0 \pm 4.8$ \\
$t$-value & 1.051 & 1.547 & 1.275 & 1.737 \\
$p$-value & 0.259 & 0.124 & 0.204 & 0.085 \\
\hline
\end{tabular}

DVH, Dose-volume histogram; MLD, mean lung dose.

have focused on monitoring the expression of certain cytokines in the course of radiotherapy to predict the occurrence of radiation pneumonia. Among them, some cytokines could predict radiation pneumonia, such as tumor necrosis factor (TNF)- $\alpha$, IL-6, IL-10, TGF-beta 1 and angiotensin-converting enzyme (ACE). Th17 and Treg cells play an important role in the inflammatory diseases (11-13), such as allergic rhinitis, bronchial asthma, rheumatoid arthritis and inflammation of the bowel. In these diseases, Th17 cells increase and Treg cells decrease; therefore, as radiation pneumonia is also an inflammation-related disease, we wished to examine whether Th17 and Treg cells are related to radiation pneumonia. In our earlier study, we established an animal model of radiation pneumonia. The results showed that unilateral lung irradiated with 20 Gy X-ray could successfully induce radiation pneumonia and Th17 cells in the blood were significantly increased. On the contrary, however, Treg cells were decreased (22). To further investigate whether Th17 and Treg cells could predict the occurrence of radiation pneumonia in patients with thoracic radiation, we collected blood samples from locally advanced esophageal cancer patients in the course of radiotherapy. This study shows that there is no significant difference in Th17 and Treg cells between the radiation pneumonia group and non-radiation pneumonia group before radiation. After the start of radiotherapy, Th17 cells increased and Treg cells decreased gradually. Until completion of radiotherapy, the difference between the two groups of Th17 and Treg cells ( $\%$ of $\mathrm{CD}^{+}$cells) was statistically significant. Therefore, we can hypothesize that Th17/Treg cells are involved in the development of radiation pneumonia. For earlier prediction of radiation pneumonia occurrence, we analyzed the changes of the ratio of Th17/Treg, with the results showing that the ratio of Th17/Treg significantly increased at the 5th week after the start of radiotherapy in the radiation pneumonia group than in the non-radiation pneumonia group. Therefore, the ratio of Th17/Treg could predict the occurrence of radiation pneumonia earlier than Th17 cells or Tregs separately.

In summary, the ratio of Th17/Treg cells is likely to be a predictor for radiation pneumonia; however, clinical trials 


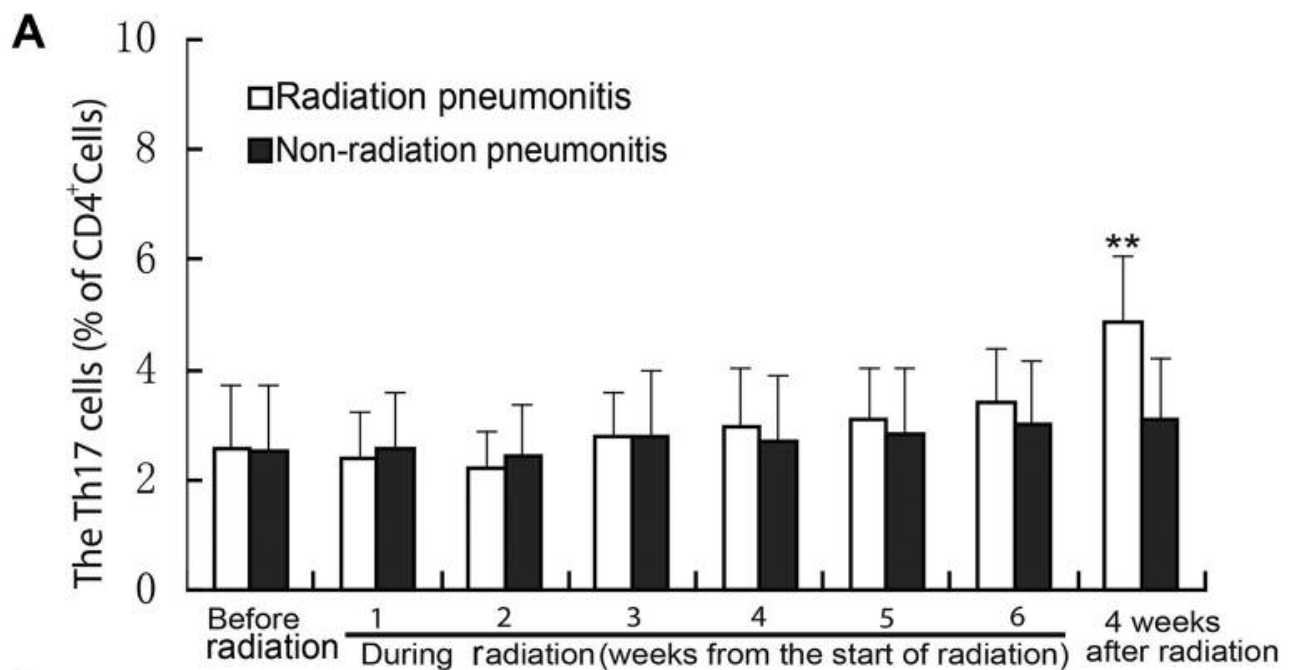

B

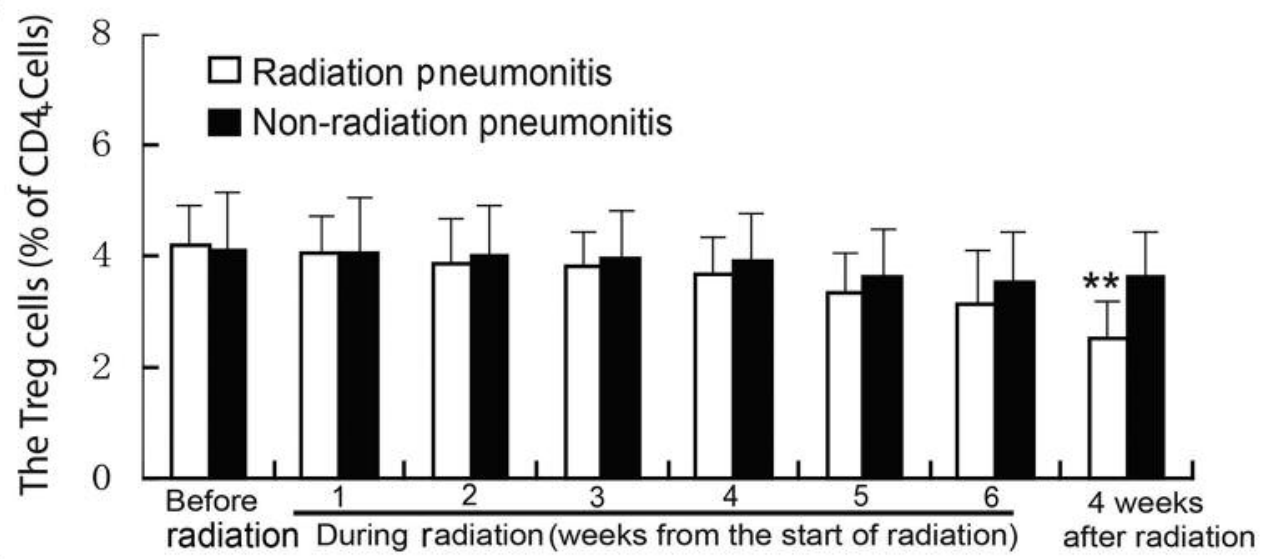

C

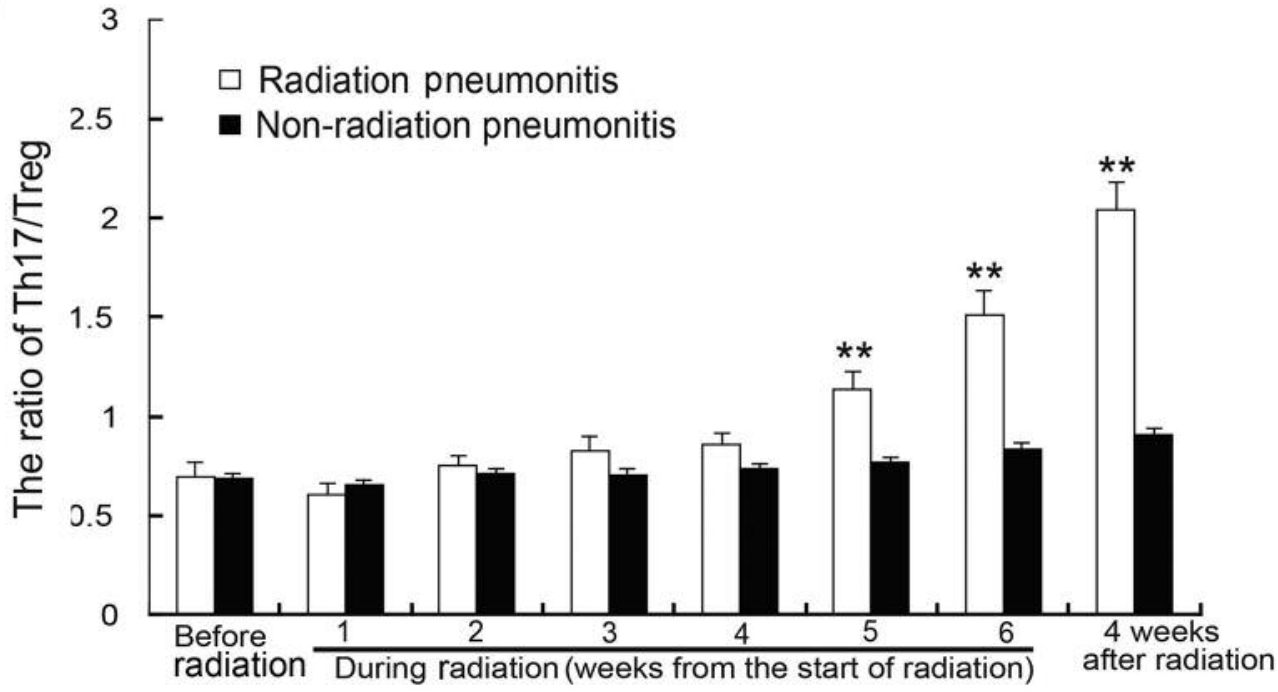

Figure 1. Relationship between Th17 cells, Treg cells, ratio of Th17/Treg and radiation pneumonia in patients with locally advanced esophageal carcinoma. Peripheral blood samples from the patients with locally advanced esophageal cancer were collected at the time point of pre-radiotherapy, $1 \mathrm{th}, 2 \mathrm{th}, 3 \mathrm{th}, 4 \mathrm{th}, 5 \mathrm{th}, 6 \mathrm{th}$ week from the radiation started and 4 weeks after radiotherapy was completed and, then, separated by density gradient centrifugation and seeded in 6-well plates at a concentration of $2 \times 10^{6} / \mathrm{ml}$. CD4 ${ }^{+} \mathrm{IL}-17^{+}$cells (Th17) and CD4+ CD25+Foxp3 cells (Tregs) were evaluated by flow cytometry analysis. A: Th17 and radiation pneumonia; B: Treg and radiation pneumonia; C: Ratio of Th17/Treg and radiation pneumonia. $*^{*} p<0.01$ vs. non-radiation pneumonia group. 
with larger samples are needed for further studies. The role of Th17/Treg cells in the incidence of radiation pneumonia and the molecular mechanisms are not yet clear, questions that will be answered with further in vivo and in vitro experiments.

\section{Acknowledgements}

This work was funded by Zhenjiang Science and Technology Development Program, No. SH2011030. We express our heartfelt thanks to all patients who participated in the study.

\section{References}

1 Fokas E, Weiss C and Rödel C: The role of radiotherapy in the multimodal management of esophageal cancer. Dig Dis 31(1): 30-37, 2013.

2 Verma V, Moreno AC and Lin SH: Advances in radiotherapy management of esophageal cancer. J Clin Med 5(10): pii: E91, 2016.

3 Shridhar R, Almhanna K, Meredith KL, Biagioli MC, Chuong MD, Cruz A and Hoffe SE: Radiation therapy and esophageal cancer. Cancer Control 20(2): 97-110, 2013.

4 Rodrigues G, Lock M, D, Souza D, Yu E and Van Dyk J: Prediction of radiation pneumonitis by dose-volume histogram parameters in lung cancer, a systematic review. Radiother Oncol 71: 127-138, 2004.

5 Arpin D, Perol D, Blay JY, Falchero L, Claude L, VuillermozBlas S, Martel-Lafay I, Ginestet C, Alberti L, Nosov D, EtienneMastroianni B, Cottin V, Perol M, Guerin JC, Cordier JF and Carrie C: Early variations of circulating interleukin-6 and interleukin-10 levels during thoracic radiotherapy are predictive for radiation pneumonitis. J Clin Oncol 23: 8748-8756, 2005.

6 Kong FM, Ao X,Wang L and Lawrence TS: The use of blood biomarkers to predict radiation lung toxicity: A potential strategy to individualize thoracic radiation therapy. Cancer Control 15: 140-150, 2008.

7 Zhao LJ, Wang LH and Wang XZ: Predictive effect of plasma TGF-beta, IL-6 and ACE level for radiation pneumonitis. Chin J Radiat oncol 15(3): 217-221, 2006.

8 Novakova-Jiresova A, Van Gameren MM, Coppes RP, Kampinga $\mathrm{HH}$ and Groen HJ: Transforming growth factor-beta plasma dynamics and post-irradiation lung injury in lung cancer patients. Radiother Oncol 71(2): 183-189, 2004.

9 Shen ZT, Shen JS, Ji XQ, Li B and Zhu XX: TGF- $\beta 1$ rs1982073 polymorphism contributes to radiation pneumonitis in lung cancer patients: A meta-analysis. J Cell Mol Med 20(12): 24052409, 2016

10 Li J, Mu S, Mu L, Zhang X, Pang R and Gao S: Transforming growth factor-beta- 1 is a serum biomarker of radiation-induced pneumonitis in esophageal cancer patients treated with thoracic radiotherapy: Preliminary results of a prospective study. Onco Targets Ther 8: 1129-1136, 2015.

11 Zhang C, Hong S and Hu G: The expression of Treg/Th17 cells related transcription factors and cytokines in PBMCs and plasma in patients with allergic rhinitis. Lin Chung Er Bi Yan Hou Tou Jing Wai Ke Za Zhi 26(5): 209-211, 2012.
12 Shi YH, Shi GC and Wan HY: Coexistence of Th1/Th2 and Th17/Treg imbalances in patients with allergic asthma. Chin Med J 124(3): 1951-1956, 2011.

13 Chao K, Zhang S, Yao J, He Y, Chen B, Zeng Z, Zhong B and Chen M: Imbalance of CD4(+)T-cell subgroups in Crohn's disease and their relationship with disease activity and prognosis. J Gastroenterol Hepatol 29(10): 1808-1814, 2014.

14 Niu X, Lu S, Li H, Liu Y, Chen Z, Zhou D, Kan M, Li Z, Yu Y, Lv $C$ and Liu S: A study of ethnic differences inTGF $\beta 1$ gene polymorphisms with the risk of radiation pneumonitis in nonsmall cell lung cancer in different ethnic study. J Thorac Oncol 7(11): 1668-1675, 2012.

15 Stenmark MH, Cai XW, Shedden K, Hayman JA, Yuan S, Ritter T, Ten Haken RK, Lawrence TS and Kong FM: Combining physical and biologic parameters to predict radiation-induced lung toxicity in patients with non-small-cell lung cancer treated with definitive radiation therapy. Int J Radiat Oncol Biol Phys 84(2): e217-222, 2012.

16 Oie Y, Saito Y, Kato M, Ito F, Hattori H, Toyama H, Kobayashi $\mathrm{H}$ and Katada K: Relationship between radiation pneumonitis and organizing pneumonia after radiotherapy for breast cancer. Radiat Oncol 8: 56, 2013.

17 Zhang XJ, Sun JG, Sun J, Ming H, Wang XX, Wu L and Chen ZT: Prediction of radiation pneumonitis in lung cancer patients: A systematic review. J Cancer Res Clin Oncol 138(12): 21032116, 2012.

18 Barriger RB, Fakiris AJ, Hanna N, Yu M, Mantravadi P and McGarry RC: Dose-volume analysis of radiation pneumonitis in nonsmall-cell lung cancer patients treated with concurrent cisplatinum and etoposide with or without consolidation docetaxel. Int J Radiat Oncol Biol Phys 78(5): 1381-1386, 2010.

19 Barriger RB, Forquer JA, Brabham JG, Andolino DL, Shapiro RH, Henderson MA,Johnstone PA and Fakiris AJ: A dosevolume analysis of radiation pneumonitis in non-small cell lung cancer patients treated with stereotactic body radiation therapy. Int J Radiat Oncol Biol Phys 1(82): 457-462, 2012.

20 Tucker SL, Jin H, Wei X, Wang S, Martel MK, Komaki R, Liu $\mathrm{HH}$, Mohan R, Chen Y, Cox JD and Liao Z: Impact of toxicity grade and scoring system on the relationship between mean lung dose and risk of radiation pneumonitis in a large cohort of patients with non-small cell lung cancer. Int J Radiat Oncol Biol Phys 77(3): 691-698, 2010.

21 Dang J, Li G, Ma L, Diao R, Zang S, Han C, Zhang S and Yao $\mathrm{L}$ : Predictors of grade $\geq 2$ and grade $\geq 3$ radiation pneumonitis in patients with locally advanced non-small cell lung cancer treated with three-dimensional conformal radiotherapy. Acta Oncol 52(6): 1175-1180, 2013.

22 Wang J, Shao G, Gong A, Jin J, Wang Y, Xue, Wu L, Wang C and Wu C: Relationship of Th17/Treg imbalance and radiation pneumonitis in rat. Jiangsu Med J 40(8): 879-881, 2014.
Received May 2, 2017

Revised May 25, 2017

Accepted May 26, 2017 